\title{
Cuento participativo en red: el reto del diálogo entre forma y contenido para obtener escenarios vibrantes
}

\author{
José María Perceval Verde ${ }^{1}$ \\ Santiago Tejedor-Calvo ${ }^{2}$
}

Recibido: 2017-09-04

Enviado a pares: 2017-09-14
Aprobado por pares: 2017-10-17

Aceptado: 2017-10-24

DOI: 10.5294/pacla.2019.22.1.3

Para citar este artículo / to reference this article / para citar este artigo

Perceval, J. M. y Tejedor-Calvo, S. (2019). Cuento participativo en red: el reto del diálogo entre forma y contenido para obtener escenarios vibrantes. Palabra Clave 22(1), e2213.

DOI: 10.5294/pacla.2019.22.1.3

\section{Resumen}

El presente artículo tiene por objeto el estudio del cuento en el ciberespacio dentro del cambio radical que suponen la comunicación virtual, la robótica y la inteligencia artificial. A partir de un enfoque metodológico de cariz teórico y mediante una exhaustiva revisión bibliográfica interdisciplinar, el trabajo reflexiona sobre las particularidades del nuevo escenario comunicativo digital y su impacto en la ideación y generación de nuevos tipos de relatos. Partimos de la hipótesis de que no existe un diálogo adecuado entre programadores informáticos, neurocientíficos y especialistas en narrativa que actúan como campos estancos y autorreferenciales que producen saberes interesantes pero paralelos. El estudio destaca que la narrativa programada utiliza solo técnicas unidireccionales de acción-respuesta propias de la psicología conductista y de una narratología exclusivamente modélica que repite sin innovar. A modo de conclusión, el trabajo incide en la

orcid.org/0000-0001-9769-7235. Universitat Autònoma de Barcelona, España. jmperceval123@gmail.com

2 orcid.org/0000-0002-5539-9800. Universitat Autònoma de Barcelona, España. santiago.tejedor@uab.cat 
necesidad de articular un marco teórico nuevo que contraste los avances y los inconvenientes de la bibliografía antropotecnológica. Por ello, la propuesta planteada propone cambiar el paradigma y aplicar las reglas derivadas de los continuadores de la escuela formalista y de los analistas de las estructuras del cuento tradicional. El artículo, como aporte original, enfatiza la necesidad de generar escenarios vibrantes mediante una apuesta creativa que introduzca en el cuento participativo en red la utilización de nuevas nociones como enjambre, inteligencia colectiva o psicología robótica para producir escenarios vibrantes. Este conjunto variado de elementos posibilita la creación de historias que potencien progresivamente la participación de los usuarios y recuperen su el rol activo.

\section{Palabras clave (fuente: tesauro de la Unesco)}

Estudios culturales; narrativas; narración de cuentos; enjambre mediático; robótica. 


\section{Participatory Storytelling Online: The Challenge of the Dialog Between Form and Content to Obtain Vibrant Settings}

\section{Abstract}

This article aims to study storytelling in cyberspace within the radical shift that virtual communication, robotics and artificial intelligence imply. Based on a theoretical methodological approach, and through a comprehensive interdisciplinary literature review, the paper reflects on the particularities of the new digital communication setting and its impact on the ideas and creation of new types of stories. We start from the hypothesis that there is no appropriate dialog among computer programmers, neuroscientists and narrative specialists who act as hermetic and self-referential fields that produce interesting but parallel knowledge. The study highlights that programmed narrative only uses one-way action-response techniques from behavioral psychology and an exclusively exemplary narratology that repeats without innovating. As a conclusion, the article emphasizes the need to construct a new theoretical framework that matches the advances and drawbacks of the anthropotechnological literature. For this, we propose to change the paradigm and apply the rules derived from the continuators of formalism and the analysts of traditional storytelling structures. The article, as an original contribution, stresses the need to create vibrant settings through a creative initiative that introduces the use of new notions such as crowdsourcing, collective intelligence or robotic psychology into participatory storytelling online. This varied set of elements enables the creation of stories that progressively enhance the involvement of users and recover their active role.

\section{Keywords (source: Unesco Thesaurus)}

Cultural studies; narratives; storytelling; media swarm; robotics. 


\section{Conto participativo em rede: 0 desafio do diálogo entre forma e conteúdo para obter cenários vibrantes}

\section{Resumo}

O presente artigo tem como objetivo estudar o conto no ciberespaço dentro da mudança radical que a comunicação virtual, a robótica e a inteligência artificial implicam. Com base em uma abordagem teórico-metodológica e através de uma exaustiva revisão bibliográfica interdisciplinar, o trabalho reflete sobre as particularidades do novo cenário da comunicação digital e seu impacto no desenho e geração de novos tipos de relatos. Partimos da hipótese de que não há diálogo adequado entre programadores de computador, neurocientistas e especialistas em narrativa que atuem como campos estanques e autorreferenciais que produzem conhecimento relevante, mas paralelo. $\mathrm{O}$ estudo destaca que a narrativa programada utiliza apenas técnicas unidirecionais de ação-resposta da psicologia comportamental e uma narratologia exclusivamente exemplar que se repete sem inovar. A título de conclusão, o trabalho enfatiza a necessidade de articular um novo marco teórico que contraponha os avanços e inconvenientes da bibliografia antropotecnológica. Por esta razão, a proposta propõe mudar o paradigma e aplicar as regras derivadas dos continuadores da escola formalista e dos analistas das estruturas do conto tradicional. $\mathrm{O}$ artigo, como uma contribuição original, enfatiza a necessidade de criar cenários vibrantes através de uma aposta criativa que introduza no conto participativo em rede a utilização de novos conceitos, como enxame, inteligência coletiva ou psicologia robótica para produzir cenários vibrantes. Esse conjunto variado de elementos permite a criação de histórias que aumentam progressivamente a participação dos usuários e recuperam seu papel ativo.

\section{Palavras-chave (fonte: tesauro da Unesco)}

Estudos culturais; narrativas; narração de histórias; exame mediático; robótica. 
Once upon a time the famous physicist Albert Einstein was confronted by an overly concerned woman who sought advice on how to raise her small son to become a successful scientist. In particular she wanted to know what kinds of books she should read to her son. Fairy Tales, Einstein responded without hesitation. Fine, but what else should I read to him after that? The mother asked. More fairy tales, Einstein stated. And after that? Even more fairy tales, replied the great scientist, and he waved his pipe like a wizard pronouncing a happy end to a long adventure ${ }^{3}$. Zdenko Zlatar

\section{Introducción: el fracaso del cuento interactivo unidireccional}

La estructura general de joven caballero enviado a una misión (objetivo/ meta, camino/obstáculos, prueba/talismán) que articula todo un grupo de relatos medievales puede considerarse como la base originaria de la narrativa de los videojuegos. La propuesta épica presenta una serie de "retos" que se ofrecen al jugador secuencialmente y que reúnen las dificultades, los objetos mágicos de apoyo taumatúrgico (amparo, protección y socorro) y los objetos trampa de ataque (desamparo, provocación y agresión). El caballero enviado a su misión debe obtener fichas, evitar obstáculos y superarlos mediante la argucia, la ayuda de los elementos mágicos adquiridos o los consejos de los personajes fantásticos con los que aprende en la ruta. Uno de los ejemplos más claros dentro del corpus de las historias populares medievales europeas se encuentra representado en la inmensa variedad de relaciones/relatos que se han establecido desde el modelo tópico del caballero/ príncipe matador de dragones (Aarne, 1910; Thompson, 1961). A través de los hermanos Grimm y su traducción mediática desarrollada por Disney, un gran número de cuentos de magia tradicionales se han convertido en hormas del conocimiento común de la realidad, y se repiten cíclicamente en la

3 Érase una vez el famoso físico Albert Einstein fue preguntado por una mujer demasiado preocupada que buscó consejos sobre cómo criar a su hijo pequeño para convertirlo en un científico exitoso. En particular, quería saber qué tipo de libros le debía leer a su hijo. Fairy tales, Einstein respondió sin dudarlo. Bien, pero ¿qué más debería leerle después de eso? La madre preguntó. Más cuentos de hadas, afirmó Einstein. ¿̨Y después de eso? Incluso más cuentos de hadas, respondió el gran científico, y agitó su pipa como un mago que pronunció un final feliz a una larga aventura.

Palabra Clave - ISSN: 0122-8285 - eISSN: 2027-534X - Vol. 22 No. 1 - Enero de 2019. e2213 
producción de la industria cultural (Hesmondhalgh, 2013). Con relación a ello, lo interesante, y a la vez decepcionante, son la pobreza y la simplificación que ha generado esta industria al abandonar la inmensa variedad y la riqueza del llamado cuento folclórico (en realidad, relato oral que incluye el mítico, el heroico y el religioso). Esta narración arquetípica ha sido fijada de forma "entomológica" por muchas de las antologías del relato "popular" (de transmisión oral) que la convierten en un objeto paralizado, arqueológico y que ocultan la continuidad y la rigidez del relato en la aparente libertad creativa que se instaura y se mitifica con el romanticismo europeo. Aunque no sea en el mismo orden ni en la misma cantidad, las 35 funciones propuestas por el estudio de Propp (1968) son la base necesaria para comenzar a plantearse una estructura formal de acción inclusiva que abarque los diferentes cuentos de magia, de animales o de seres fantásticos. La acusación de etnocentrismo e incluso de "arianismo" del modelo de Propp ha sido superada por los defensores de su universalidad (Dundes, 1964), por los que siguen la narrativa estructural de Greimas (1983), por los partidarios de la complicación/resolución de Labov (1972), por los que diferencian el modelo como Todorov (1965) como por las actuales aportaciones realizadas en el terreno de la narratología por los estudios japoneses de la narrativa samurái o los indostánicos sobre los grandes ciclos de Mahabbarata y Ramayana que influyen tanto en los relatos de Bollywood como en Stars Wars (Zlatar, 1997; Piantelli, 1997; Sreenivas, 2010; Nusz, 2012; Jai$\mathrm{dka}, 2016)$. En este sentido, lo importante que plantea el modelo proppiano no es señalar tanto la meta con el "héroe/heroína" (obsesionante en las estructuras neoliberales), sino estructurar la vía de acceso que permite una visión mucho más empática y participativa. En definitiva: la emoción de la aventura antes que la perspectiva del premio.

Jaidka (2016) parte de la idea de que las historias tienen dos tipos de enclave: uno en un tiempo y un lugar concreto analizable diacrónicamente y otro como parte del patrimonio mundial que puede responder a un análisis sincrónico transnacional. Dentro del "arte" de la narración, propone la construcción de puentes para resaltar las similitudes subyacentes a la literatura mundial reelaborando la idea de cosmopolitismo cultural relacionado con el ser humano creativo, un antiguo ideal indio de Vasudhaiva Kutum- 
bakam (que concibe el mundo como el hogar) o africano bantú de Ubuntu (que propone una idea de sociedad abierta a todos los vientos). Los clásicos de la épica hindú, como el Panchatantra ${ }^{4}$ que ha trabajado Alemany (2007), el Kathasaritsagar ${ }^{5}$ estudiado ampliamente por Serebriakov (1880) y las noches árabes se relacionan con las nuevas épicas de la era de la tecnología de la información. Las historias que se escuchan y repiten en la tradición oral comparten ciertas cadencias que se extienden a través de las fronteras del tiempo y del espacio superando la brecha entre personas sin minusvalorar la riqueza y la variedad del mundo representado.

\section{El cuento como un juego coral y horizontal}

¿Cómo insertar creativamente esta nueva dinámica en el cuento participativo en red? La oposición principal se plantea entre la estructura lineal del relato, como un camino (on the road), y la circular de la parábola o el cuento de moraleja, aunque con estructuras mixtas e híbridas de gran riqueza. El "modelo búsqueda" y el "modelo pregunta" articularían una nueva relación entre el cuento tradicional y la parábola, quizá en la línea del escritor italiano Gianni Rodari (Salvadori y Asteriti, 2002), que recupera el poder revolucionario y transgresor de las antiguas fábulas. Las otras oposiciones formales son perfectamente universalizables como las divisiones hombre/mujer, joven/adulto y alto/bajo podrían ser complementadas por otras polaridades y divisiones: hombre/dios, humanos/animales, seres humanos/extraterrestres. Estas divisiones cada vez se han tornado más híbridas subsumiéndose en la categoría transversal de "seres con poderes" que las unifica, sean varón/mujer/animal/ser fantástico o incluso personajes que traspasan géneros o identidades. Del mismo modo, los modelos culturales se hibridan en "relatos mix". Se trataría de lo que Sidhar (2009) denomina borrowings (mezcla de apropiación, reescritura y transformaciones que son transducciones) con respecto a la cultura de Bollywood. Semiología y narratología se han dedicado a glosar, explicar, aclarar y definir la estructura de los cuentos al mismo tiempo que establecían normas para su ideación. Lo importante en esta nueva etapa de creación cultural, no es solo

4 Colección de fábulas en idioma sánscrito, en prosa y verso, compuesto después del siglo III a. C. y que se atribuyen a Vishnu Sharma.

5 Compilación de mitos, leyendas populares, fábulas y cuentos de hadas indios. 
la interpretación (que resulta igualmente necesaria y pertinente), sino disponer de herramientas para los creadores y establecer una serie de normas de señalización para el flujo de los materiales creativos que funcionen dialógicamente. Contra la idea romántica del creador aislado e inspirado por la divinidad, contra la idea igualmente romántica del relato como una emanación de las raíces de pueblos míticos (base de la antropología nacionalista), hay que pensar en estructuras comunes al creador y en elementos accesibles mediante el aprendizaje. Al sistematizar, no se elimina la elección del narrador ni, en absoluto, su libertad, sino que, al contrario, se le potencia al proporcionarle nuevas herramientas creativas. Como indica Berger (2003), es posible que Durkheim haya muerto y que nos encontremos en una etapa cercana a Sherlock Holmes, o incluso, quizá, también en los caminos de la serendipia o serendipity (Merton y Barber, 2004) aplicados al juego educativo por Kickmeeier-Rust y Dietrich (2009) desde el punto de vista del diseño. Como señala Bal (2009), lo que no pertenece a la visión del detective se debe al destino accidental y a la serendipia; esto es, el camino racional, intuitivo o del azar. El cuento participativo es aquel que rompe la jerarquía unidireccional, la pirámide del narrador modelo, para convertirse en una obra coral y horizontal, donde los aportes, no solo cambian el juego, sino que son el juego. El análisis de las narrativas es la mejor manera de obtener una buena comprensión de cómo se organizan e integran los diversos aspectos de la información humana en la construcción de nuestra realidad. Es necesario construir un nuevo modelo dialógico entre narrativa y tecnología. Los estudios más dinámicos vienen de los enfoques computacionales y cognitivos de la narratología que vinculan las tecnologías de la información y la comunicación con la narrativa y el proceso de generación de historias desarrollando interesantes campos creativos, como los ensayos recopilados por Ogata y Akimoto (2016). En sus propuestas, unen los trabajos sobre inteligencia artificial y la neurociencia o ciencia cognitiva con el fin de diseñar la narratología. La intención es desarrollar sistemas que automáticamente generen, creen o produzcan contenidos explicativos digitales relacionados con una variedad de géneros narrativos existentes o futuros (sistemas de procesamiento de imágenes y películas de línea argumental abierta). Asimismo, la aplicación de la robótica por parte de las investigadoras finlandesas Peltonen y Wickström (2014) al espacio educativo me- 
diante el uso de juegos de computadora con fines pedagógicos es una idea fascinante que se está haciendo cada vez más popular entre los formadores, los investigadores y los desarrolladores. Se abordan, de este modo, desafíos fundamentales para la investigación, tales como métodos para facilitar el diseño del juego de aprendizaje o la individualización de las experiencias lúdicas, la personalización educativa y el diseño de juegos emergentes (Kickmeier-Rust y Dietrich, 2009). La aplicación de los modelos narrativos a la inteligencia artificial ha sido objeto de estudio de los trabajos de Gervás, Peinado, Lönneker-Rodman y Meister (2006), quienes concluyen que una combinación metodológica de descripción, análisis y generación automática de historias tiene el potencial de un avance cualitativo mutuamente beneficioso en la investigación de modelos narrativos. Lo que favorecen estas nuevas herramientas metodológicas y fácticas son los "escenarios vibrantes", aquellos en que la empatía y el entusiasmo confluyen en un ritmo poderoso donde el lector se siente tanto partícipe como creador.

\section{Modelos formales de relato abierto de ficción}

El denominado transmedia storytelling representa un proceso en el que elementos integrales de una ficción se dispersan sistemáticamente a través de múltiples canales de distribución con el propósito de crear una experiencia de entretenimiento unificada y coordinada. Idealmente, cada medio hace su propia contribución única al desarrollo de la historia (Jenkins, 2007, 2011). Más allá del componente meramente técnico, el artículo sigue las recomendaciones de Snydery Waite (2010) y concibe que la transversalidad de soportes y de relatos solo resulta posible mediante la aplicación de reglas formales. La vuelta al formalismo permite un diálogo más útil y equilibrado en que los dos partenaires - creador y receptor - se encuentran en un mismo plano dialógico en lugar de verse sometidos a un monólogo improductivo debido a los imperativos técnicos (o tecnoideológicos) de la unidireccionalidad. Los teóricos actuales se concentran en aspectos sociales, contextuales y performativos del significado, uniéndolos a la idea de que cada representación es inherentemente multimodal y multisemiótica. El trabajo de los semióticos como Halliday (2014) ha sido la principal referencia para nuestra comprensión de dinámicas significantes complejas involucradas en distintos medios. En este sentido, los conocimientos generados por tal trabajo, cuyo principal 
objetivo es determinar la necesidad de discusión de modelos, acorde con las diferentes recetas metodológicas (con los cambios y las adaptaciones necesarias), podrían ser de utilidad para la investigación sobre los aspectos contextuales, performativos y multimodales de la significación del cuento popular, como nos señalan múltiples autores de una u otra manera (Duggan, Haase y Callow, 2016; Baldock, 2006; Haase, 2008; Tomashevski, 1965). Esto, claro es, teniendo en cuenta los préstamos culturales (cultural borrowings) de apropiación, reelaboración y transformación de formas y temáticas en el proceso de transducción. Lo interesante es que los cuentos rusos analizados por Propp ya eran en sí mismos un modelo híbrido de translación y transliteratura al recoger la tradición occidental heroica, la de los modelos de las estepas (especialmente, en su vertiente chamánica) y la de los kanatos musulmanes (genios y yinns del mundo islámico). Existe, por supuesto, un arte del narrador, concebido como la capacidad de generar narraciones (mensajes) basadas en la estructura (código). Este arte corresponde a la noción de rendimiento (ofperformance) definida por Chomsky (1999), y se aleja de la noción de genio autoritario, concebido románticamente como un secreto personal, difícilmente explicable que ya Barthes (1975) criticaba. El modelo de Propp (1984) se basa en un análisis de un centenar de textos de la colección Afanasiev que se incluían en la subcategoría de los cuentos de hadas. La extensión a otras recopilaciones se ha producido desde las colecciones nórdicas (con la escuela finlandesa de estudios folclóricos) a las indias del Pantchatantra (Krohn, 1971; Holbek, 1992) basada en el trabajo de Aarne (1910) con un índice tipo, que más tarde fue revisado por Thompson (1961).

A pesar de que Arvidsson (2005) discute la universalidad de las 31 funciones de Propp, reconoce con Berger (1997) que estas se pueden encontrar, no solo en los cuentos populares que Propp analizó, sino también en el núcleo del conjunto de las narraciones, pudiendo extenderse incluso al modelo de videojuegos propio de la cultura popular de comienzos del siglo XXI (Berger, 2002). Con relación a ello, destaca del legado de Propp la forma de hacer realidad los sueños (Zipes, 2007). Sin embargo, considerado como un hito del análisis semiológico y concebido como la base de la narratología formal, la crítica lo ha convertido en un templo lleno de tabúes 
que se sacraliza o se critica laicamente que incluye el problema añadido de todos los estudios comparatistas que diseccionan elementos estructurales, caracteres y esferas de acción (Arvidson, 2005). El planteamiento de Propp se ha mantenido ante los embates críticos, fundamentalmente, por una regla que es clave en el cuento popular (el relato que recoge la memoria en las sociedades preindustriales), y es que todos responden a una estructura formal que no los constriñe, sino que modula la narración. Este modelo de Propp es una herramienta útil para el análisis descontextualizado de las narrativas, independiente del contexto social e histórico donde están insertas y donde se utilizan, con fines ideológicos y sociales concretos. La "narrativa per se" no es un arma contra la narrativa, sino una explicación de su ágil manejo por creadores individuales, que sin ella no podrían ser comprendidos por sus propias sociedades. Resulta, por tanto, necesario diseccionar dos tipos de contenidos formales para su utilización: por un lado, los de la horma, la forma de relato articulado por Propp; por otro, la mixidad de contenidos, los personajes emblemáticos que permiten construir los relatos mediante la empatía del lector hacia modelos de maldad, bondad o aventura conocidos y manejables. A ello se suma una serie de escenarios perfectamente reconocibles, ambientes (tormenta, páramo desértico, bosque profundo, cruce de caminos, etc.) que han sido organizados por Thompson (1961). No obstante, la mixidad actual llevaría a unir determinados personajes como "bruja” y "hada" (en alemán son denominadas con el mismo término, hegen) como las mujeres de enormes poderes (mucho mayores que los de los varones en toda la tradición universal y que puede utilizar para el bien o para el mal). Cuando Zipes (2006) desmenuza el cuento de hadas, cada migaja se convierte en un relato en sí mismo. Personajes, motivos e incidentes revelan su autonomía creativa: “Tenemos al simplón que resulta finalmente ser notablemente astuto, el hijo más joven que es oprimido por sus hermanos o su propio padre, la hermosa pero maltratada hija menor y el soldado explotado por sus superiores"6 (pp. 4-5). Contamos, de este modo, con personajes, ambientes y contextos que son elementos ineludibles y maravillosos para el proceso creativo. El dinamismo vendrá de las oposiciones y de las conjunciones, menos señaladas en la narratología tradicional, así como de la elasticidad de la sucesión de

6 Las traducciones son nuestras. 
situaciones que se encadenan secuencialmente en los ambientes que escenifican las situaciones más asombrosas. En otras palabras, el cuento de la magia europea obtiene su fuerza narrativa de las oposiciones de hombres y mujeres, jóvenes y adultos, rey y campesinos (ricos y pobres) que se convierten en temas de interés. La bruja tiene como contrapartida el hada; el tirano, al rey bondadoso; el avaro, al rico generoso; los animales siempre tienen dos caras. Se trata de un sistema de oposiciones que debe ser ágil, pero no excluyente, y donde no se ha de tener miedo a la utilización de malvados, porque el mal es necesario. En ese sistema de oposiciones, hay que redefinir a la mujer y al héroe que han sido transformados por las revoluciones cortesanas, burguesas y fascistas. Holbek (1986) analiza el cambio de rol de la mujer que ha sido demonizada o convertida en víctima por la reapropiación burguesa de los narradores masculinos, especialmente, a partir de los Grimm, para ser liberada en los cuentos de género actuales que rompen con la idea de la pareja como una mera recompensa final y la convierten en un conjunto simpático y empático de colaboración mutual. Del mismo modo, la conquista de la fama se transforma en el reconocimiento por el valor de los cuentos originales. Holbek clasifica los diferentes modelos de "patrones de héroes" según lo propuesto por Campbell (1993) y Von Hahn, Rank, Raglan y De Vries (Holbek, 1986). Estos eruditos elaboraron, independientemente unos de otros, propuestas de patrones estructurales en cuentos míticos y heroicos. De este modo, constata que las diferencias y las similitudes se basan en "el material básico común" de los relatos universales. Hay que superar la revolución aristocrática (cuento cortesano) y burguesa (héroe individual neoliberal) para volver al relato empático y comunitario original. De particular interés son las opiniones de Vries sobre la comparación de cuentos heroicos y cuentos de hadas: "Las diferencias principales son que los elementos maravillosos desempeñan un papel importante en los cuentos de hadas y uno menor en cuentos heroicos y que los cuentos de hadas invariablemente tienen finales felices mientras que los cuentos heroicos terminan trágicamente” (Holbek, 1986, p. 330).

\section{Narradores, contextos y momentos}

Mito, historia y leyenda dan sentido a una sociedad al contar su origen fundacional. Hay que liberar el cuento heroico de la narrativa nacionalista que 
se lo apropió para justificar la aparición del estado o la individualista burguesa que lo sitúa como el líder de la empresa común. De este modo, es posible afirmar que el cuento heroico cuenta con parecidas reglas narrativas al cuento de hadas.

No existe un modelo narrativo estructural subyacente a todas las narrativas complejas. Existe, sin embargo, una panoplia de elementos que se combinan constantemente en la actividad de los bardos/"griots" o "djelis” (narradores de historias de África Occidental) a nivel universal. Por ello, se plantea la necesidad de añadir un estudio sobre narradores con idiosincrasias diferentes en contextos y momentos distintos para, de este modo, alcanzar una recuperación de lo verbalizado frente a lo textual. Del mismo modo, se debe incorporar la nueva corriente de los estudios folclóricos que distinguen las diferencias regionales, el estudio de las idiosincrasias personales y la adaptación a cada situación contextual y con diferentes públicos (Bauman, 1986). Frente a la escuela estrictamente lingüística (Tomashevski, 1965), se trabaja sobre niveles verbalizados que incluyen los estados de ánimo o las señales interpretativas (Bal, 2009). Este paso nos permite un análisis en red que debería ampliar el patrón inmanente que se encuentra detrás de cada narrativa. Se trataría de no quedarse en la aplicación fácil del tipo (se repiten los esquemas de Blancanieves, Cenicienta [Rooth, 1951] o la Bella Durmiente, entre otros). La glocalización aplicada a la narrativa permite la ágil relación entre las estructuras formales del relato, las transducciones de otros contextos, las adaptaciones y las relaciones transculturales, sin caer en una pretensión de autenticidad inexistente ni en un globalismo vacío. Del mismo modo, el diálogo entre los géneros diversos permite una transmedialidad e intermedialidad ágil. Para ello, se necesita la participación de los públicos como actores y no como simples receptores. Pero ¿cómo lograrlo?

\section{De los ataques de la imprenta al cuento oral a la creación en escenarios empáticos}

El bardo no es exactamente un memorión, pues, aunque sigue unas reglas nemotécnicas, ni tan siquiera repite el mismo relato cada vez que cuenta un 
hecho. En cada ocasión, adapta el mensaje a su público y a la circunstancia concreta (tiempo y espacio en que despliega su obra de narrador). En el momento en que el bardo habla, canta, grita, se emociona y llora, el público sufre un proceso de hipnosis al ritmo y cadencia de sus palabras: se realiza una precisa interacción entre el fluido del habla del narrador y la mente de los receptores que terminan siendo parte de la coreografía de sus palabras como un coro griego. El cuentista se adapta a la recepción que espera y pacta cada relato con su público. Al mismo tiempo, la estructura de lo que expresa puede atenerse a normas muy estrictas que el público conoce y que favorecen la buena comunicación. Son hormas que se llenan de los más diversos contenidos según la habilidad y la capacidad del narrador (Aarne, 1961; Thompson, 1961). Los asistentes no presencian algo "teatral" en el sentido de falso. Un cuento es aquello que nos contamos. Un cuento no es una mentira, siempre es una verdad en alguna forma. Creado para solucionar nuestros miedos, ordena el caos en un coherente relato con personajes que pueden ser héroes con poderes sobrenaturales, fuerzas naturales con aspecto y sentimientos humanos, animales que hablan o personas que tienen el poder de hablar con animales. A través de sus maravillosas aventuras, el problema situado en ese mundo fantástico queda solucionado. La tradición oral mantendrá con toda su fuerza el relato empático durante milenios. Su coherencia se basará en dos aspectos fundamentales: la existencia de hormas trabajadas y perfeccionadas que permanecen intactas y que, al mismo tiempo, se aplican a situaciones concretas, variables, en cada momento. La Cenicienta podría ser un ejemplo perfecto de narración que se repite continuamente, igual, aunque siempre diferente, en la versión cinematográfica que Hollywood nos ofrece cada año. La cultura de lo escrito mantendrá también durante siglos una fluida relación con lo oral e igualmente dará la primacía a los textos sagrados. Estos conservarán su legitimidad porque fueron verbalizados en algún momento por la revelación divina. Los relatos de la cultura oral pasarán a la tradición escrita en las hagiografías de los santos y en las crónicas de los reyes. El poder aprovechará el cuento para contarse a sí mismo, para narrar lo legítimo de su presencia. La imprenta, sin embargo, representa una innovación paralizadora, ya que fija e inmoviliza la cultura oral. Los letrados, además, sitúan el relato oral en un apartado exterior y secundario, ahora definitivamente convertido en lo que conocemos como "cuento" (es decir, un relato 
fantasioso y mentiroso), ubicado en el lado infantil y femenino, en el lado de la incultura y el saber popular.

La imprenta conservó muchos relatos orales al imprimirlos, pero estos perdieron su fuerza. La magia del cuento oral se diluía, ya que no era algo que se contaba sino que se leía. La decadencia del relato oral y de la transmisión oral tendrá un largo recorrido con luchas y resistencias. Se sufrirá una larga desvalorización de lo oral desde la cultura de lo impreso. Finalmente, acabará domesticada y mitificada, como folclore o manifestación del supuesto espíritu del pueblo, tan cercano al nacionalismo del siglo XIX. Los ataques de la imprenta al cuento oral fueron de varios tipos: al fijarlo, este pierde la adaptabilidad que tenía a diversas circunstancias y momentos. Los elementos carnavalescos e irónicos desaparecen, así como su función crítica. En la corte, los cuentos se llenan de princesas y príncipes para justificar una clase social que estaba a punto de ser superada por la burguesía. En el ambiente burgués, la moral y la moralina se imponen y eliminan el erotismo, lo malsonante, lo escatológico, incluso lo "terrorífico", tan habitual en los cuentos de advertencia. De los hermanos Grimm a Disney, los cuentos originales son prácticamente asesinados para ofrecernos cadáveres gloriosos. Contradictoriamente, los formalistas, como Propp o Thompson, restauraron la idea de que el cuento era una horma y debía estar vivo. Posteriormente, la materia de los cuentos se rebeló en el cine juvenil de terror. La industria cultural, como señala Hesmondhalgh (2013), es una sucesión de bardos que construyen historias y una serie de gestores que organizan el escenario para representarlas; los bardos actuales son, según Monaci (2016), las plataformas de cocreación. En este sentido, los estudios se han dirigido, básicamente, a la comprensión del mecanismo de cogeneración y asegurar la calidad y la fluidez de la participación en los procesos de cogeneración de contenidos digitales, al tiempo que investigaban la evaluación de la calidad de Wikipedia, uno de los proyectos de conocimiento abierto más conocidos de internet (Monaci, 2008).

\section{Relatos y redes de artefacto-actor}

Una serie de trabajos han presentado la base tecnológica y la arquitectura como un vehículo para modelar, obtener y almacenar información de los 
usuarios en el ciberespacio, centrándose en las llamadas "redes de artefacto-actor". Se trata de la combinación de redes sociales clásicas con redes de artefactos construidas para el uso de los objetos de información y sus respectivas conexiones (Reinhardt, Varlemann, Matthias y Wilke, 2010). De la misma forma, las normas de cortesía estudiadas en la relación lingüística se están aplicando a la verbalización de instrucciones por parte de los robots. Las máquinas que necesitan ofrecer recomendaciones a los usuarios pueden mitigar el tono dominante adoptado a menudo con el uso de una base de recursos derivados de la literatura de cortesía y fórmulas del lenguaje informal. De este modo, confieren opciones al discurso de ayuda mediante fórmulas o marcadores que reducen el aspecto unidireccional de las "órdenes" y las transforman en consejos y conversaciones que establecen el espacio virtual. Los avances en la psicología robótica aplicada a la narrativa y a la estética, desarrollados en Japón en el Robot Actor Project, por el investigador de artes escénicas Zaven Paré junto al profesor Ishiguro Hiroshi de la Universidad de Osaka y el Instituto Internacional de Estudios Superiores de Kioto han mostrado la posibilidad de un diverso (universo paralelo) en el que trabajar nuevas formas narrativas (Paré, 2016). El estudio de Torrey, Fussell y Kiesler (2012) demostró que los avances en la robótica permiten a los robots ayudar con el uso del lenguaje natural de cortesía, aunque el campo de la HRI (human-robot interaction) aún no ha desarrollado una estrategia de comunicación para dar consejos de manera efectiva. Se plantea la necesidad de estudiar la morfología humana para la aplicación robótica no invasiva. Del mismo modo, la utilización de fórmulas propias de la comunicación visual se aplica a la robótica como la altura del hablante, su forma de hablar, la intensidad y moldeabilidad de la voz, entre otros.

\section{Noción de enjambre (swarm media) como nuevo modo de relación mediática}

El enjambre de partículas canónicas es descrito por Kennedy (2007) como un algoritmo estocástico que optimiza los resultados al estudiar la población basándose en principios sociopsicológicos. El enjambre no utiliza la selección previa permitiendo a todos los miembros de la población pervivir desde el comienzo de un juicio hasta su final, ayudando con sus inte- 
racciones a una mejora iterativa de la calidad de los problemas a lo largo del tiempo. Un vector numérico de dimensiones $\mathrm{D}$, usualmente inicializado al azar en un espacio de búsqueda, se conceptualiza como un punto en un sistema de coordenadas cartesianas de alta dimensión. El punto se describe como una partícula debido a su movilidad en el espacio que prueba nuevos valores del parámetro. En la medida en que realizan este comportamiento simultáneamente y tienden a agruparse en regiones óptimas del espacio de búsqueda, se les conoce como "enjambre de partículas". Los investigadores del campo de la inteligencia colectiva (Rosenberg, Baltaxe y Pescetelli, 2016) han demostrado que los grupos pueden superar a las personas al tomar decisiones, predicciones y pronósticos. Sin embargo, los métodos estadísticos tradicionales, e ideológicamente conformados por el individualismo neoliberal, tratan a la población como una multitud de agentes independientes que proporcionan a los "analizadores” información aislada en forma de encuestas y acciones individuales en las transacciones de mercado. Este sistema ha demostrado una utilidad relativa en análisis de mercado y fracasos notables en los análisis políticos a partir de encuestas electorales unipersonales (el resultado no era del grupo analizado sino del grupo formado por el encuestador y los encuestados, por lo cual se había roto un grupo y creado artificialmente otro). La eficacia relativa de estos sistemas (de muchedumbre) contrasta con la realidad de los sistemas naturales (de grupo) que aprovechan la inteligencia compartida. En el mundo natural, los grupos forman sistemas de bucle cerrado en tiempo real, enjambres que convergen en soluciones de sincronía. Los trabajos de las investigadoras Pata (2011) y Phillips (2012) describen un experimento de diseño participativo influenciado por la actividad de enjambre del que se deriva un nuevo enfoque para escribir narrativas en las comunidades de aprendizaje virtual de la denominada web social, que contrasta con los enfoques tradicionales de narración de cuentos. Esta planificación incluiría la utilización de herramientas de software libre, tales como microblogging, repositorios sociales de imágenes, ubicaciones mapeadas en la ciudad y narradores libres. De este modo, recreó un enjambre en un ecosistema con nuevos formatos narrativos en evolución dentro de este espacio híbrido. De este modo, es posible señalar un enfoque hacia el escenario de los cuentistas, bardos o "griots" en un nuevo ecosistema híbrido y transmedial (Perceval, 2015). 


\section{Hacia un nuevo ecosistema híbrido de relatos dinámicos}

La desaparición, que no dilución, de la individualidad en internet coincide con el aumento de la creatividad en el anonimato de las redes, pero también con una neurosis propia del aislamiento individual ante la máquina que es la multitud de espectadores no presentes y fantasmagóricos (Perceval, Simelio y Forga, 2013; Stewart, 2014). La función de un relato participativo en este espacio neblinoso, como un bosque de Brocelianda, donde todo está, pero nada se ve, se centra en rescatar el dinamismo y la magia de la creación del cuento/relato oral interrumpido por la etapa de la imprenta (la llamada era Gutenberg).

En África Occidental, provenientes de la tradición del imperio mandinga (siglo XIII), aún quedan "griots" o "djeli" que actúan como contadores de historias que forman una casta normalmente hereditaria y endogámica, rodeada de particulares tabúes y prevenciones por su poder mágico. Este poder viene de una propiedad del lenguaje que sigue siendo actual: el lenguaje hace vivir lo que relata (una imagen perfecta para el mundo virtual). En el animismo de estas sociedades, no hay una frontera estricta entre pasado, presente y futuro, como tampoco existe en internet. Incluso la adivinación se trata como un "recuerdo del futuro". Por tanto, los tiempos, los lugares y los personajes se mezclan en el relato del cuentista y se convierten en elementos intercambiables y adaptables a cualquier situación (Perceval y Tejedor, 2006). Vellar (2009) describe en su trabajo sobre la industria cultural y el público participativo nuevas formas de cocreación que están diseñando lo que ella llama "escenarios vibrantes", donde la producción industrial converge con la producción de iguales (los pares), integrando la colaboración abierta entre los usuarios con formas de organización que permiten la mejora del contenido creativo. Las industrias culturales definidas por Hesmondhalgh (2013) cambiarían su estrategia piramidal de la época de la imprenta por la nueva estructura horizontal de no anárquicos miembros de un club de intereses comunes. Esta estructura necesita más que nunca normas y señalizaciones para que el fluido cultural circule libremente. La historia narrada y asumida (storytelling) puede utilizarse en todos los esce- 
narios probables y algunos improbables de la dinámica de la comunicación actual (Madsen y Nielsen, 2009).

En el campo interdisciplinario de los estudios de ciencia y tecnología, se introdujo el concepto de affordances (ofrecimientos) como una forma de navegar la Scylla y Charybdis del determinismo tecnológico, esto es, romper este mundo separado y contradictorio: por un lado, nuevos modos de relaciones sociales activamente causados por formas particulares de la tecnología y por el construccionismo tecnológico; por otro, los artefactos tecnológicos socialmente moldeados tanto en forma como en significado (Hutchby, 2001). En contraste, se articula una teoría de affordances como ecología perceptiva (Gibson, 1979), que considera las tecnologías como artefactos que "pueden ser formados y conformados por las prácticas que los seres humanos usan en la interacción con, alrededor y a través de ellos” (Hutchby, 2001, p. 444). Este autor define específicamente las affordances como "aspectos funcionales y relacionales que enmarcan, sin determinar, las posibilidades de una acción auténtica en relación con un objeto" (Hutchby, 2001). Gibson (1979), por su parte, acuñó el término para indicar la relación epistémica entre el agente y su medio. Este intercambio aparece cuando el agente detecta una información específica que le permite modular su acción. Se trata, en definitiva, del establecimiento de un diálogo entre diversos actores que crean y gestionan los relatos para convertirlos en obras de una industria cultural renovada. Para ello, se necesita superar el modelo etnocéntrico mediante modelos glocalizadores, el sistema jerárquico unidireccional autoritario mediante el rediseño del "héroe/protagonista" (masculino o femenino) dotado de superpoderes y aristocrático e individualista en un personaje empático y modélico e invertir la apropiación nacionalista y exclusivista mediante modelos híbridos culturalmente. En conclusión, la clave de todo el edificio es la redefinición de "agon" platónico (entre forma y relato), evitando las trampas de considerar la forma como un adorno o complemento, ya que es la esencia definitoria de la narrativa. La forma (ahora unida a la creación de software) es la que canaliza el género, la figura, el estilo, la que libera el texto individual de la sacralidad del autor/genio, la que permite conectar las hormas intemporales con las vicisitudes de la historia concreta y actual. Este trabajo incide en la necesidad de intervenir en el 
diálogo comercial, en el cual la ingeniería robótica, la inteligencia artificial y la neurociencia trabajan conjuntamente con el objetivo de generar nuevos modelos para el mercado. La creación de historias puede servirse de estas herramientas y de estos avances para convertirlos en elementos liberadores que faciliten la participación de los diferentes actores para volver a contar con un público activo y participativo.

\section{Referencias}

Aarne, A. (1910). Verzeichnis der Märchenypen. Helsinki: Suomalainen Tiedeakatemia.

Aarne, A. (1961). The types of the folktale: A classification and bibliography. Helsinki: The Finnish Academy of Science and Letters.

Alemany, J. (2007). Panchatantra. Barcelona: Paidós.

Arvidsson, A. (2005). Computer games as fiction and social interaction. Umeå: Umeå Universitet.

Bal, M. (2009). Narratology: Introduction to the theory of narrative. Toronto: University of Toronto Press.

Baldock, P. (2006). The place of narrative in the early years curriculum: How the tale unfolds. Nueva York: Routledge.

Barthes, R. (1975). The pleasure of the text. Nueva York: Hill\&Wang.

Bauman, R. (1986). Story, performance, and event: Contextual studies of oral narrative. Nueva York: Cambridge University Press.

Berger, A. (1997). Narratives in popular culture, media, and everyday life. Thousand Oaks: Sage.

Berger, A. (2002). Video games: A popular culture phenomenon. New Brunswick: Transaction. 
Berger, A. (2003). Durkheim is dead! Sherlock Holmes is introduced to sociological theory. San Francisco: Altamira Press/Rowman \& Littlefield.

Campbell, J. (1993). The hero with a thousand faces. Londres: Fontana Press.

Chomsky, N. (1999). Ideas and ideals. Cambridge: Cambridge University Press.

Duggan, A., Haase, D. y Callow, H. (2016). Folktales and fairy tales: Traditions and texts from around the world. Santa Barbara: ABC-CLIO.

Dundes, A. (1964). The morphology of north American indian Folktales. Helsinki: Suomalainen Tiedeakatemia.

Gervás,P.,Peinado,F.,Lönneker-Rodman,B.yMeister,J.(2006).Narrativemodels: Narratology meets artificial intelligence. En International Conference on Language Resources and Evaluation. Satellite Workshop: Toward ComputationalModelsofLiteraryAnalysis (pp.44-51). Génova:LREC.

Gibson, J. (1979). The theory of affordances. Nueva Jersey: Hilldale.

Greimas, A. (1983). Structural semantics: An attempt at a method. Lincoln: University of Nebraska Press.

Haase, D. (ed.) (2008). The Greenwood Encyclopedia of Folktales and Fairy Tales. Londres: Greenwood Press.

Halliday, W. (2014). Folklore and Mythology. Indo-European Folk-Tales and Greek Legend. Cambridgeshire: Cambridge University Press.

Hesmondhalgh, D. (2013). Cultural industries. Londres: Sage.

Holbek, B. (1986). Interpretation of fairy tales. Helsinki: Suomalainen Tiedeakatemia.

Holbek, B. (1992). On the comparative method in folklore research. Turku: Nordic Institute of Folklore. 
Hutchby, I. (2001). Technologies, texts and affordances. Sociology, 35(2), 441-456. DOI: $10.1017 /$ S0038038501000219

Jaidka, M. (2016). Narratives across borders. Newcastle: Cambridge Scholars Publishing.

Jenkins, H. (2007). Convergence culture: Where old and new media collide. Nueva York: New York University Press.

Jenkins, H. (2011, julio 31). Transmedia 202: Further reflections. Recuperado de http://henryjenkins.org/2011/08/defining_transmedia_ further_re.html

Kennedy, J. (2007). Particle swarm optimization. Encyclopedia of Machine Learning, 1(1), 760-766. DOI: 10.1007/978-0-387-30164-8_630

Kickmeier-Rust, M. D. y Dietrich, A. (2009). Emergent design: Serendipity in digital educational games. Graz: University of Graz.

Krohn, K. (1971). Folklore methodology. Austin: University of Texas Press.

Labov, W. (1972). Sociolinguistic patterns. Philadelpia: University of Pennsylvania Press.

Madsen, S. y Nielsen, L. (2009). Exploring persona-scenarios-using storytelling to create design ideas. En Human work interaction design: Usability in social, cultural and organizational contexts (pp. 57-66). Berlín: Springer.

Merton, R. K. y Barber, E. (2004). The travels and adventures of serendipity: A study in sociological semantics and the sociology of science. Nueva Jersey: Princenton University Press.

Monaci, S. (2008). Quality contents creation in a commons: Based peer production on line environment: The IT. Wikipedia Experience. 
En IADIS International Conference WWW/Internet (pp. 11-18). Friburgo: IADIS.

Monaci, S. (2016). Boosting creativity through digital disruption? The role of co-creation platforms in the media production field. Westminster Papers in Communication and Culture, 11(1), 49-64. DOI: $10.16997 /$ wpcc. 218

Nusz, A. (2012). The foundational structures behind Star Wars (Tesis de maestría, University of Louisville, Kentuchy, Estados Unidos).

Ogata, T. y Akimoto, T. (2016). Computational and cognitive approaches to narratology. Hersehey: IGI Global.

Paré, Z. (2016). L'Âge d’or de la robotique Japonaise. París: Les Belles Lettres.

Pata, K. (2011). Participatory design experiment: Storytelling Swarm in hybrid narrative ecosystem. En B. K. Daniel (ed.), A handbook of research on methods and techniques for studying virtual communities: Paradigms and phenomena (pp. 482-508). Nueva York: Hershey Information Science Reference.

Peltonen, M. y Wickström, M. (2014). 3D-prints and robots play a part in my story: Participatory learning action and content creation in a library maker space. En Libraries for children and young adults with literacy and reading. Lyon: IFLA WLIC.

Perceval,J. M. (2015). Historia mundial de la comunicación. Madrid: Cátedra.

Perceval, J. M. y Tejedor Calvo, S. (2006). El cuento multimedia interactivo. Comunicar, 26, 177-182. Recuperado de https://www.redalyc. org/articulo.oa?id $=15802627$

Perceval, J. M., Simelio, N. y Forga, M. (2013). Yo, tú, él: la desaparición de los pronombres en internet. Hacia la personalidad múltiple en 
red. En F. Sabés y J. J. Verón (eds.), Comunicación y la red: nuevas formas de periodismo (pp. 231-238). Huesca: Asociación de Periodistas de Aragón.

Phillips, A. (2012). A creator's guide to transmedia storytelling: How to captivate and engage audiences across multiple platforms. Nueva York: McGraw-Hill.

Piantelli, M. (1997). About the Ramayana Structure: Epics and fable. En Proceedings of the Ninth International Ramayana Conference (pp. 253-263). Turín: International Ramayana Conference.

Propp, V. (1968). Morphology of the folktale. Austin: University of Texas Press.

Propp, V. (1984). Theory and history of folklore. Manchester: Manchester University Press.

Reinhardt, W., Varlemann, T., Matthias, M. y Wilke, A. (2010.) Modeling, obtaining and storing data from social media tools with Artefact-Actor-Networks. Paderborn: University of Paderborn.

Rooth, A. (1951). The cinderella cycle. Lund: Gleerups.

Rosenberg, L., Baltaxe, D. y Pescetelli, N. (2016). Crowds vs swarms, a comparison of intelligence. Swarm/Human Blended Intelligence Workshop. DOI: 10.1109/SHBI.2016.7780278

Salvadori, M. L. y Asteriti, A. (2002). Apologizing to the ancient fable: Gianni Rodari and his influence on italian children's literature. Baltimore: Johns Hopkins University Press.

Serebriakov, I. (1880). A few thoughts on the Katha Sarit Sagara. Nueva Delhi: Munshiram Manoharlal. 
Sidhar Wright, N. (2009). “Tom Cruise? Tarantino? E.T.? ... Indian!” Innovation through imitation in the Cross-cultural Bollywood Remake. Recuperado de http://clublum.com/images/Scope-Cultural Borrowings_Final\%20clublum.pdf\#page $=206$

Snyder, P. C. y Waite, J. (2010). Using transmedia storytelling techniques to invigorate graphic communications programs. Visual Communications Journal, 50(1), 24-31. Recuperado de http://gceaonline.org/ publications/visual-communications-journal/

Sreenivas, D. (2010). Structure of Narratives: Applying Propp's folktale morphology to entertainment-education films. México: University of New Mexico.

Stewart, K. G. (2014). Blogs, books, \& breadcrumbs: A case study of transmedial fairy tales. Provo: Brigham Young University.

Thompson, S. (1961). The types of the folktale: A classification and bibliography. Helsinki: Academia Scientiarum Fennica.

Todorov, T. (1965). Teoría de la literatura, textos de los formalistas rusos. Buenos Aires: Signos.

Tomashevski, B. (1965). Thematics. En L. T. Lemon y J. R. Marion (eds.), Russian formalist criticism: Four essays. Lincoln: University of Nebraska Press.

Torrey, C., Fussell, S. y Kiesler, S. (2012). How a robot should give advice. En Proceedings of the 8th ACM/IEEE International Conference on Human-Robot Interaction. Tokio: IEEE Press.

Vellar, A. (2009). Le industrie culturali ei pubblici partecipativi: dalle comunità di fan ai social media. Roma: Aracne.

Zipes, J. (2002). Breaking the magic spell: Radical theories of folk and fairy tales. Kentucky: University Press of Kentucky. 
Zipes, J. (2006). Fairy tales and the art of subversion. Abingdon: Routledge.

Zipes, J. (2007). When dreams came true: Classical fairy tales and their tradition. Nueva York: Routledge.

Zlatar, Z. (1997). Approaches to the Ur-Mahabharata. Sydney Studies in Society and Culture, 15, 243-284. Recuperado de https://openjournals.library.sydney.edu.au/index.php/SSSC/article/view/8011 